\title{
A numerical scheme for axisymmetric solutions of curvature-driven free boundary problems, with applications to the Willmore flow
}

\author{
UWE F. MAYER ${ }^{\dagger}$ \\ 1955 Missouri St., San Diego, CA 92109, USA \\ AND \\ GIERI SIMONETT \\ Department of Mathematics, Vanderbilt University, Nashville, TN 37240, USA
}

[Received 21 May 2001 and in revised form 2 October 2001]

\begin{abstract}
We present a numerical scheme for axisymmetric solutions to curvature-driven moving boundary problems governed by a local law of motion, e.g. the mean curvature flow, the surface diffusion flow, and the Willmore flow. We then present several numerical experiments for the Willmore flow. In particular, we provide numerical evidence that the Willmore flow can develop singularities in finite time.
\end{abstract}

Keywords: Willmore flow; numerical solutions; singularities

\section{Introduction}

In this paper we construct a numerical method for axisymmetric solutions for some curvature-driven geometric evolution problems. These moving boundary problems arise from models in physics, the material sciences, or differential geometry. Although our main focus will be on the Willmore flow, our numerical method is presented in a more general context that applies to other problems. The situation we consider is the following. Let $\Gamma_{0}$ be a compact closed immersed orientable surface in $\mathbb{R}^{3}$. A curvature-driven geometric evolution problem consists of finding a family $\Gamma=\{\Gamma(t): t \geqslant 0\}$ of smooth closed immersed orientable surfaces in $\mathbb{R}^{3}$ which evolve according to a law

$$
V=f\left(\kappa_{1}, \kappa_{2}\right), \quad \Gamma(0)=\Gamma_{0} .
$$

Here $V$ denotes the normal velocity of $\Gamma$, while $\kappa_{1}, \kappa_{2}$ are the principal curvatures of $\Gamma(t)$. The mean curvature is given through $H=\frac{1}{2}\left(k_{1}+k_{2}\right)$, and the Gauss curvature through $K=k_{1} k_{2}$. We denote the Laplace-Beltrami operator of $\Gamma(t)$ by $\Delta$. One obtains the mean curvature flow with this notation by setting $f=-H$, the surface diffusion flow by setting $f=\Delta H$, and the Willmore flow by setting

$$
f=\Delta H+2 H\left(H^{2}-K\right) .
$$

Throughout this paper we assume the free boundary to be an orientable connected compact axisymmetric two-dimensional surface $\Gamma(t)$ immersed in $\mathbb{R}^{3}$. We assume that the evolution is

Email: mayer@math.utah.edu

Email: simonett@math.vanderbilt.edu 
sufficiently smooth, that is, for small enough time $\tau$ we have

$$
\Gamma(t+\tau)=\left\{y \in \mathbb{R}^{3}: y=x+\rho(x, t, \tau) N(x, t), x \in \Gamma(t)\right\}
$$

for some smooth function $\rho$ and unit (outer) normal $N$ of $\Gamma(t)$. Let $V(x, t)=\left.\frac{\partial \rho}{\partial \tau}(x, t, \tau)\right|_{\tau=0}$ denote the normal velocity of $\Gamma(t)$. Then we will consider evolution laws of the form

$$
V(x, t)=f\left(\kappa_{1}(t), \kappa_{2}(t)\right)(x),
$$

where $f$ is a local operator. The signs are chosen in such a way that a sphere has positive curvature with respect to the outer normal. By the definition of $V$ a family of expanding spheres has positive normal velocity.

Our approach leads to a first-order-accurate backward Euler time discretization, with a spatial discretization needed only of a generating curve of the axisymmetric surface. Due to the local character of the evolution, the matrices involved in the computations explained below are in fact matrices that have only entries on the main diagonal and on the two neighboring (modulo $n$ ) diagonals. Such an $n \times n$ matrix can be inverted very fast; one needs $\mathrm{O}(n)$ operations. This is very different from the general three-dimensional case without symmetry, compare $[15,16]$. To validate our numerical scheme we consider the test problem of a sphere evolving according to the mean curvature flow, one of the rare cases where an exact solution is known. The findings indicate that the proposed scheme is highly accurate, allowing for numerical simulation of the evolution up to the time of singularity.

As the Willmore flow is our principal example, we list some of the known results relating to this flow. The following result concerning existence, regularity, and uniqueness of solutions has been recently established in [23].

\section{THEOREM}

(a) Suppose $\Gamma_{0}$ is a compact closed immersed orientable $C^{2, \alpha}$-surface in $\mathbb{R}^{3}$. Then the Willmore flow admits a unique family $\Gamma:=\{\Gamma(t): t \in[0, T]\}$ of smooth surfaces $\Gamma(t)$ which evolve according to (1), (2).

(b) If $\Gamma_{0}$ is sufficiently close to a sphere in the $C^{2, \alpha}$-topology, then the solution exists globally and converges exponentially fast towards a sphere.

We also refer to [12] for related results concerning global existence of solutions.

Having pointed out that solutions close to spheres converge to spheres then raises the question about existence and stability of additional equilibria. In general, any equilibrium of (1), that is, any closed smooth surface that satisfies the equation

$$
\Delta H+2 H\left(H^{2}-K\right)=0
$$

is called a Willmore surface [27: p. 282]. Willmore surfaces arise as the critical points of the functional

$$
W(\phi):=\int_{\phi(M)} H^{2} \mathrm{~d} S,
$$

see [24] or [27: Section 7.4]. Here, $\phi: M \rightarrow \mathbb{R}^{3}$ is a smooth immersion of $M$ into $\mathbb{R}^{3}$. This functional was proposed first around the year 1810 by Sophie Germain to model the elastic energy 
of an elastic surface, and enjoyed only periodic interest in the mathematical community until Willmore's work in the mid-1960s brought it back to the forefront of mathematical research [25-27].

The question of the limiting surfaces for the Willmore functional can then be rephrased as a variational problem: given a smooth closed orientable surface $M_{g}$ of genus $g$ determine the infimum $W\left(M_{g}\right)$ of $W(\phi)$ over all immersions $\phi: M_{g} \rightarrow \mathbb{R}^{3}$ and classify all manifolds $\phi\left(M_{g}\right)$ which minimize $W$. It is known that $W(M) \geqslant 4 \pi$ for any surface $M$ and that the minimum is attained if and only if $\phi(M)$ is embedded as a round sphere [27: Theorem 7.7.2]. A characterization of all Willmore immersions $\phi: S^{2} \rightarrow \mathbb{R}^{3}$ has been obtained in [5]; the possible values of $W(\phi)$ are $4 \pi n$ where $n=1$, or $n \geqslant 4$ even, or $n \geqslant 9$ odd. For the torus $T^{2}$ there is the long-standing 'Willmore Conjecture' asserting that $W\left(T^{2}\right)=2 \pi^{2}$. The conjecture has been proved for some conformal classes $[14,18]$. The conjecture is also known to be true for surfaces whose images under stereographic projection are surfaces of revolution in $\mathbb{R}^{3}$ [13]. In particular, the value $2 \pi^{2}$ is attained by those tori of revolution whose generating circles have ratio $1 / \sqrt{2}$, see for instance [27: p. 274]. It is known that there exist embedded Willmore surfaces in $\mathbb{R}^{3}$ of arbitrary genus. Such surfaces arise, for instance, as the images of embedded minimal surfaces in $S^{3}$ under stereographic projection of $S^{3}$ into $\mathbb{R}^{3}$. Examples of compact embedded Willmore surfaces that are not stereographic projections of compact embedded minimal surfaces in $S^{3}$ were first found in [20]. Lastly we mention that all surfaces of genus $g$ that are absolute minimizers of the functional $W$ are necessarily embedded as Willmore surfaces. The existence of a minimizer for any $g \geqslant 1$ follows from the work of Kusner [10] and Simon [22]. We refer to $[21,22,27]$ and the references therein for more details and interesting results.

The Willmore flow is the $L^{2}$-gradient flow for the functional (5) on the moving boundary, see for example [11], and also [15] for related work on gradient flows. Thus the Willmore flow has the distinctive property that it evolves surfaces in such a way as to reduce the total quadratic curvature (also called the Willmore energy). More precisely, let $\Gamma_{0}$ be any surface that is $C^{2, \alpha}$-smooth. Then

$$
\int_{\Gamma(t)} H^{2}(t) \mathrm{d} \mu \leqslant \int_{\Gamma_{0}} H^{2}(0) \mathrm{d} \mu, \quad 0 \leqslant t \leqslant T,
$$

where $[0, T]$ denotes the interval of existence guaranteed in the existence theorem quoted before, and where $H(t)$ denotes the mean curvature of $\Gamma(t)$. This gradient flow structure has been employed in [9] by using Brakke's Surface Evolver (see [4]) to compute numerical solutions for minimizers of the Willmore functional. Their approach is to start with polyhedral surfaces with very few vertices, then to use the numerical evolution and to refine the triangulation as needed to finally approach a numerically smooth limiting surface. Our focus here is not so much on the limiting surfaces, but on the geometric behavior of solutions for various initial geometries. Because of the use of the axisymmetry, we need considerably less computational effort to simulate a smooth flow.

It is interesting to compare the Willmore flow with the surface diffusion flow given by $f=\Delta H$. In the latter case it is well known that hypersurfaces evolve in such a way as to reduce surface area, while preserving the volume enclosed by $\Gamma(t)$, see [6] for instance. It is therefore of interest to compare and contrast the behavior of solutions for the Willmore flow and the surface diffusion flow, as will be done in some of the subsequent numerical simulations.

Our numerical simulations include the evolution of a torus of revolution, of a dumbbell surface, of an erythrocyte-shaped surface, of a cylinder of revolution, and of an immersed surface obtained by revolving parts of a limaçon curve around an axis. All these examples reveal interesting, new properties of the Willmore flow. For instance, we establish numerically that the Willmore flow drives dumbbell surfaces to a sphere, no matter how small the neck is. This is in strict contrast 
to the surface diffusion flow which seems to lead to a pinch-off, see [16]. Our experiment with an erythrocyte-shaped surface indicates that the Willmore flow can lose embeddedness, that is, can drive embedded surfaces to self-intersections. This property is shared by the surface diffusion flow, but clearly not by the mean curvature flow, where the maximum principle prevents self-intersections from forming. It is well known that the mean curvature flow preserves convexity. Our numerical simulation based on a cylindrical surface seems to indicate otherwise for the Willmore flow, as is the case for the surface diffusion flow.

It is an open question whether or not the Willmore flow can develop singularities in finite time. Our last example in Section 8.5 based on a limaçon curve provides numerical evidence that this can indeed happen. We therefore conjecture that the Willmore flow can develop singularities in finite time.

Conjecture There exists a family $\Gamma:=\{\Gamma(t): t \in[0, T)\}$ of smooth compact closed immersed orientable surfaces in $\mathbb{R}^{3}$ which evolve according to the Willmore flow given by (1), (2) such that the limiting surface $\Gamma(T)$ is singular, where $T<\infty$.

\section{Discretization}

The proposed scheme for the moving free boundary problems involves essentially two steps. First discretize in time, and then discretize the surface to deal with the spatial variables. We assume that the considered surfaces are sufficiently smooth in both time and space, so that at least all derivatives occurring in the following computations exist and are continuous. This is in particular known to be true for the solutions to the Willmore flow (see [23]), which is our main application.

\subsection{Finite differences}

The normal velocity is the temporal derivative of the surfaces $\Gamma(t)$ in the normal direction and thus the first-order approximation of (3) is given by the finite difference equation

$$
N(x, t) \cdot \frac{\Gamma(x, t+h)-\Gamma(x, t)}{h}=f\left(\kappa_{1}(t+h), \kappa_{2}(t+h)\right)(x) .
$$

As the right-hand side is evaluated at the next time step, this is a first-order-accurate backward Euler time discretization.

One should actually write $\widetilde{\Gamma}$ and so on because the above equation describes approximate solutions; by a similar abuse of notation we will call the left side of the above equation $V(x, t)$ again and hence

$$
\begin{aligned}
\Gamma(x, t+h) & =\Gamma(x, t)+h V(x, t) N(x, t), \\
V(x, t) & =f\left(\kappa_{1}(t+h), \kappa_{2}(t+h)\right)(x) .
\end{aligned}
$$

Finally, approximate the dependence of the curvature of the next time step by

$$
\kappa_{i}(x, t+h) \approx \kappa_{i}(x, t)+h\left(L_{i} V\right)(x, t)
$$

where $L_{i}, i=1,2$, is a linear operator which is formally defined via

$$
\left(L_{i} V\right)(x, t)=\left.\frac{\mathrm{d}}{\mathrm{d} h} \kappa_{i}(\Gamma(x, t)+h V(x, t) N(x, t))\right|_{h=0} .
$$


We use Taylor's expansion to obtain the first-order approximation

$$
\begin{aligned}
f\left(\kappa_{1}(t+h), \kappa_{2}(t+h)\right) \approx & f\left(\kappa_{1}(t), \kappa_{2}(t)\right) \\
& +D_{1} f\left(\kappa_{1}(t), \kappa_{2}(t)\right)\left(\kappa_{1}(t+h)-\kappa_{1}(t)\right) \\
& +D_{2} f\left(\kappa_{1}(t), \kappa_{2}(t)\right)\left(\kappa_{2}(t+h)-\kappa_{2}(t)\right),
\end{aligned}
$$

and thus (omitting the arguments for brevity)

$$
\left.\left.f\right|_{t+h} \approx\left(f+h D_{1} f L_{1} V+h D_{2} f L_{2} V\right)\right|_{t} .
$$

Writing $D f=\left(D_{1} f, D_{2} f\right)$ and $L=\left(\begin{array}{l}L_{1} \\ L_{2}\end{array}\right)$, we arrive at the first-order approximation

$$
V=f+h D f L V
$$

or equivalently,

$$
(\text { id }-h D f L) V=f \text {. }
$$

Note that for this semi-implicit scheme only the dependency of $f$ with regard to $V$ at the next time step is linearized; we still use the full non-linear $f$ for evaluation at the current time.

\subsection{Spatial discretization}

The surface $\Gamma$ is generated by rotation of a curve $\gamma$. The curve will be subdivided into a finite collection of disjoint arcs $\gamma_{i}$. Choose points $z_{i} \in \gamma_{i}$; we write $x_{i}$ and $y_{i}$ for the $x$ - and $y$-coordinate of $z_{i}$, respectively.

We assume $f$ to be a local operator. Hence for $m=1,2$ there are numbers $s_{i j}^{m}$ associated with $D_{m} f$ (possibly depending locally on the current position and shape of $\gamma$ ) so that for any suitable function $w$ one has

$$
\left(D_{m} f\left(\kappa_{1}, \kappa_{2}\right) w\right)\left(z_{i}\right) \approx \sum_{j=i-1}^{i+1} s_{i j}^{m} w\left(z_{j}\right) .
$$

In order to keep the notation simple we have suppressed the time variable $t$ in our notation. Note that we have restricted to local operators $f$ so that $D_{m} f$ can be approximated at any given point by only using data at that point and at the immediate neighbors. This is why the sum above runs from $i-1$ to $i+1$. To give a specific example, let us consider the function $f$ of (2) which can also be written as

$$
f\left(\kappa_{1}, \kappa_{2}\right)=\frac{1}{2} \Delta\left(\kappa_{1}+\kappa_{2}\right)+\frac{1}{2}\left(\kappa_{1}+\kappa_{2}\right)\left(\kappa_{1}-\kappa_{2}\right)^{2} .
$$

In this case one obtains

$$
D_{m} f\left(\kappa_{1}, \kappa_{2}\right) w=\frac{1}{2} \Delta w+\frac{1}{2}\left(\kappa_{1}-\kappa_{2}\right)^{2} w+(-1)^{m-1}\left(\kappa_{1}+\kappa_{2}\right)\left(\kappa_{1}-\kappa_{2}\right) w .
$$

In the same fashion, since $L_{m}$ from (7) is a local linear mapping as well, we have

$$
\left(L_{m} V\right)\left(z_{i}\right) \approx \sum_{j=i-1}^{i+1} l_{i j}^{m} V\left(z_{j}\right)
$$


for some coefficients $l_{i j}^{m}$ depending on $\Gamma(t)$ but independent of $V(x, t)$. Finally, we obtain a linear system for the unknown velocity

$$
\forall i: \quad \sum_{j=i-2}^{i+2}\left(\delta_{i j}+\sum_{k=i-1}^{i+1} h\left(s_{i k}^{1} l_{k j}^{1}+s_{i k}^{2} l_{k j}^{2}\right)\right) V\left(z_{j}, t\right)=f\left(\kappa_{1}(t), \kappa_{2}(t)\right)\left(z_{i}\right) .
$$

The evaluation of $f$ in the right-hand side here is assumed to be of local character. That is, it might need more data than just evaluation at the point $z_{i}$, but no more than the data at $z_{i}$ and the two neighboring points. Other than this there is no restriction on $f$, provided the general assumptions of the set-up are satisfied. Finally, the sum in the left-hand side runs from $i-2$ to $i+2$ because of the product of two sums inside the parentheses.

\section{Approximation of the curvature}

\subsection{Approximation of the first principal curvature}

The curvature is considered to be located at the vertices. The motivation of the formula used is as outlined below. Our surface is given through rotation of a curve $\gamma$. The curvature of this curve is of course a principal curvature of the surface. There is a well known formula for the change of the length $\mathcal{L}$ of a simple curve under normal variations $\phi v$, with $v$ the outer unit normal of $\gamma$,

$$
\delta \mathcal{L}(\gamma, \phi):=\left.\frac{\mathrm{d}}{\mathrm{d} \tau} \mathcal{L}(\gamma+\tau \phi v)\right|_{\tau=0}=\int_{\gamma} \kappa_{1} \phi \mathrm{d} s
$$

where $\kappa_{1}$ is the signed curvature of the positively oriented curve. Let $\eta_{\epsilon}$ be a piecewise linear function of one real variable with $\eta_{\epsilon}(0)=1$ and $\eta_{\epsilon}(t)=0$ for $|t|>\epsilon$. Pick a point $z$ on a smooth curve $\gamma$ embedded in $\mathbb{R}^{2}$ and consider the variation of $\gamma$ with variation function $\phi_{\epsilon}=\eta_{\epsilon}(|\cdot-z|)$ supported on an $\epsilon$-interval about $z$. Then by (12)

$$
\delta \mathcal{L}\left(\gamma, \phi_{\epsilon}\right)=\int_{\mathrm{d} I_{\epsilon}(z)}\left(1-\frac{1}{\epsilon}|x-z|\right) \kappa_{1}(x) \mathrm{d} s_{x} \approx 2 \kappa_{1}(z) \int_{0}^{\epsilon}\left(1-\frac{\rho}{\epsilon}\right) \mathrm{d} \rho=\epsilon \kappa_{1}(z) .
$$

On the discretized curve the same effect of this variation can be achieved by the (normal) movement of one vertex. We choose $\epsilon$ from above to be the average distance from $z_{i}$ to its two neighbors. Consider the length of the discretized curve as a function of the position of $z_{i}$, while all other vertices are fixed: that is, consider the function

$$
\mathcal{L}_{i}(z)=C+\left|z_{i+1}-z\right|+\left|z-z_{i-1}\right|
$$

Replacing just one of the vertices $z_{i}$ by $z_{i}+\tau v_{i}$ (index $i$ fixed, vector $v_{i}$ fixed) leads to

$$
\left.\frac{\mathrm{d}}{\mathrm{d} \tau} \mathcal{L}_{i}\left(z_{i}+\tau v_{i}\right)\right|_{\tau=0}=\left(\frac{z_{i}-z_{i-1}}{\left|z_{i}-z_{i-1}\right|}-\frac{z_{i+1}-z_{i}}{\left|z_{i+1}-z_{i}\right|}\right) \cdot v_{i}
$$

One can now immediately read off the gradient of $\mathcal{L}_{i}$ as being

$$
\nabla \mathcal{L}_{i}:=\nabla \mathcal{L}_{i}\left(z_{i}\right)=\frac{z_{i}-z_{i-1}}{\left|z_{i}-z_{i-1}\right|}-\frac{z_{i+1}-z_{i}}{\left|z_{i+1}-z_{i}\right|} .
$$


Recall that $\nabla \mathcal{L}_{i}\left(z_{i}\right)$ points in the direction of the largest increase of $\mathcal{L}_{i}$. On the other hand, it is easy to see that the biggest increase of $\mathcal{L}_{i}$ occurs for variations in normal direction of $z_{i}$. We conclude that the unit outer normal $v_{i}$ of the curve at the vertex $z_{i}$ is given by

$$
v_{i}= \pm \nabla \mathcal{L}_{i} /\left|\nabla \mathcal{L}_{i}\right|
$$

The sign in (16) is ' + ' if $\nabla \mathcal{L}_{i}$ points towards the outside of $\gamma$, and '-' otherwise. From (13)-(15), together with our choice of $\epsilon$, we obtain the approximation

$$
\kappa_{1}\left(z_{i}\right) \approx \kappa_{1 i}:=\frac{\nabla \mathcal{L}_{i} \cdot v_{i}}{\left(\left|z_{i}-z_{i-1}\right|+\left|z_{i+1}-z_{i}\right|\right) / 2} .
$$

If $\nabla \mathcal{L}_{i}$ happens to be zero we see that the two vectors $z_{i+1}-z_{i}$ and $z_{i}-z_{i-1}$ are parallel, and we normalize one of them and turn it clockwise by $90^{\circ}$ to get the outside unit normal, and in this case we have of course $\kappa_{1}\left(z_{i}\right)=0$.

The starting and the end points of the curves are on the coordinate axes if we do not have a closed loop. In particular, the starting point $z_{0}$ is always on the $x$-axis in this case. We can define for computations' sake $z_{-1}=\left(x_{1},-y_{1}\right)$, and compute all the expressions as outlined before. In particular

$$
\kappa_{1}\left(z_{0}\right) \approx \kappa_{10}:= \pm \frac{\left|\nabla \mathcal{L}_{0}\right|}{\left|z_{0}-z_{1}\right|}
$$

where

$$
\nabla \mathcal{L}_{0}=\frac{z_{0}-z_{-1}}{\left|z_{0}-z_{-1}\right|}-\frac{z_{1}-z_{0}}{\left|z_{1}-z_{0}\right|}=\frac{2 z_{0}-z_{1}-z_{-1}}{\left|z_{1}-z_{0}\right|} .
$$

The formulae above of course also apply with suitably changed indices for the last point $z_{n-1}$, which is also on a coordinate axis in case we do not have a loop. Similarly, we can take advantage of possible symmetry to the $y$-axis, and then need only half the computational effort for the simulations. This reasoning also applies throughout in the following sections.

\subsection{Approximation of the second principal curvature}

We assume that the curve under consideration is rotated about the $x$-axis, positively oriented, and parametrized by arc length, that is, we have a parametrization $s \mapsto(x(s), y(s))$. Then the second principal curvature is given by

$$
\kappa_{2}(s)=-\frac{x^{\prime}(s)}{y(s)}
$$

provided $y(s) \neq 0$, and $\kappa_{2}(s)=\kappa_{1}(s)$ if $y=0$ (see the Appendix). As mentioned before, we write $x_{i}$ for the $x$-coordinate of the vertex $z_{i}$, etc. The derivative is approximated as usual by a finite difference,

$$
x_{i}^{\prime} \approx \frac{1}{2} \cdot\left(\frac{x_{i+1}-x_{i}}{\left|z_{i+1}-z_{i}\right|}+\frac{x_{i}-x_{i-1}}{\left|z_{i}-z_{i-1}\right|}\right)
$$

As mentioned before, the starting and the end points of the curves are on the coordinate axes if we do not have a closed loop, and in this case we have $\kappa_{2}=\kappa_{1}$. 


\section{Linearization of the curvature}

\subsection{Linearization of the first principal curvature}

Use $V_{i}$ to denote the velocity of the vertex $z_{i}$. In this section we compute coefficients $l_{i j}^{1}$ such that for the operator $L_{1}$ as defined in (7) we have

$$
L_{1} V\left(z_{i}, t\right) \approx \sum_{j} l_{i j}^{1} V_{j}
$$

We use (17). We write $D_{i}=\left(\left|z_{i+1}-z_{i}\right|+\left|z_{i}-z_{i-1}\right|\right) / 2$. Let tildes denote quantities referring to the configuration at the next time step. In particular, the vertices are given by $\tilde{z}_{i}=z_{i}+h V_{i} v_{i}$ and the first principal curvature is given by $\tilde{\kappa}_{1 i}=\left(\nabla \tilde{\mathcal{L}}_{i}\left(\tilde{z}_{i}\right) \cdot \tilde{v}_{i}\right) /\left(\tilde{D}_{i}\right)$, compare with Section 3.1. Also, $\nabla \tilde{\mathcal{L}}_{i}\left(\tilde{z}_{i}\right) \cdot \frac{\mathrm{d}}{\mathrm{d} h} \tilde{v}_{i}=0$ because $\tilde{v}_{i}$ is a unit vector and $\nabla \tilde{\mathcal{L}}_{i}\left(\tilde{z}_{i}\right)$ is parallel to $\tilde{v}_{i}$. Hence we define the coefficients $l_{i j}^{1}$ via

$$
\sum_{j} l_{i j}^{1} V_{j}=\left.\frac{\mathrm{d}}{\mathrm{d} h} \tilde{\kappa}_{1 i}\right|_{h=0}=\frac{\left.\frac{\mathrm{d}}{\mathrm{d} h}\left(\nabla \tilde{\mathcal{L}}_{i}\left(\tilde{z}_{i}\right)\right)\right|_{h=0} \cdot v_{i}}{D_{i}}-\left.\frac{\mathcal{L}_{i} \cdot v_{i}}{D_{i}^{2}} \cdot \frac{\mathrm{d}}{\mathrm{d} h} \tilde{D}_{i}\right|_{h=0} .
$$

Starting out with

$$
\nabla \tilde{\mathcal{L}}_{i}\left(\tilde{z}_{i}\right)=\frac{z_{i}+h V_{i} v_{i}-z_{i-1}-h V_{i-1} v_{i-1}}{\left|z_{i}+h V_{i} v_{i}-z_{i-1}-h V_{i-1} v_{i-1}\right|}-\frac{z_{i+1}+h V_{i+1} v_{i+1}-z_{i}-h V_{i} v_{i}}{\left|z_{i+1}+h V_{i+1} v_{i+1}-z_{i}-h V_{i} v_{i}\right|},
$$

standard computations lead to

$$
\begin{aligned}
\frac{\mathrm{d}}{\mathrm{d} h}\left(\nabla \tilde{\mathcal{L}}_{i}\left(\tilde{z}_{i}\right)\right) & \left.\right|_{h=0} \cdot v_{i}=\left(\frac{\left(\left(z_{i}-z_{i-1}\right) \cdot v_{i}\right)\left(\left(z_{i}-z_{i-1}\right) \cdot v_{i-1}\right)}{\left|z_{i}-z_{i-1}\right|^{3}}-\frac{v_{i-1} \cdot v_{i}}{\left|z_{i}-z_{i-1}\right|}\right) V_{i-1} \\
& +\left(\frac{1}{\left|z_{i}-z_{i-1}\right|}+\frac{1}{\left|z_{i+1}-z_{i}\right|}-\frac{\left(\left(z_{i}-z_{i-1}\right) \cdot v_{i}\right)^{2}}{\left|z_{i}-z_{i-1}\right|^{3}}-\frac{\left(\left(z_{i+1}-z_{i}\right) \cdot v_{i}\right)^{2}}{\left|z_{i+1}-z_{i}\right|^{3}}\right) V_{i} \\
& +\left(\frac{\left(\left(z_{i+1}-z_{i}\right) \cdot v_{i}\right)\left(\left(z_{i+1}-z_{i}\right) \cdot v_{i+1}\right)}{\left|z_{i+1}-z_{i}\right|^{3}}-\frac{v_{i+1} \cdot v_{i}}{\left|z_{i+1}-z_{i}\right|}\right) V_{i+1}
\end{aligned}
$$

Similarly, as

$$
\tilde{D}_{i}=\frac{1}{2}\left(\left|z_{i}+h V_{i} v_{i}-z_{i-1}-h V_{i-1} v_{i-1}\right|+\left|z_{i+1}+h V_{i+1} v_{i+1}-z_{i}-h V_{i} v_{i}\right|\right)
$$

we obtain

$$
\begin{aligned}
\left.\frac{\mathrm{d}}{\mathrm{d} h} \tilde{D}_{i}\right|_{h=0}= & -\frac{\left(z_{i}-z_{i-1}\right) \cdot v_{i-1}}{2\left|z_{i}-z_{i-1}\right|} V_{i-1} \\
& +\left(\frac{1}{2}\left(\frac{z_{i}-z_{i-1}}{\left|z_{i}-z_{i-1}\right|}-\frac{z_{i+1}-z_{i}}{\left|z_{i+1}-z_{i}\right|}\right) \cdot v_{i}\right) V_{i}+\frac{\left(z_{i+1}-z_{i}\right) \cdot v_{i+1}}{2\left|z_{i+1}-z_{i}\right|} V_{i+1}
\end{aligned}
$$


Grouping terms according to $V_{i-1}, V_{i}$, and $V_{i+1}$ finally leads to expressions for $l_{i, i-1}^{1}, l_{i i}^{1}$, and $l_{i, i+1}^{1}$, while all the others are zero,

$$
\begin{aligned}
l_{i, i-1}^{1}= & \frac{1}{D_{i}}\left(\frac{\left(\left(z_{i}-z_{i-1}\right) \cdot v_{i}\right)\left(\left(z_{i}-z_{i-1}\right) \cdot v_{i-1}\right)}{\left|z_{i}-z_{i-1}\right|^{3}}-\frac{v_{i-1} \cdot v_{i}}{\left|z_{i}-z_{i-1}\right|}+\kappa_{1 i} \frac{\left(z_{i}-z_{i-1}\right) \cdot v_{i-1}}{2\left|z_{i}-z_{i-1}\right|}\right), \\
l_{i i}^{1}= & \frac{1}{D_{i}}\left(\frac{1}{\left|z_{i}-z_{i-1}\right|}+\frac{1}{\left|z_{i+1}-z_{i}\right|}-\frac{\left(\left(z_{i}-z_{i-1}\right) \cdot v_{i}\right)^{2}}{\left|z_{i}-z_{i-1}\right|^{3}}\right. \\
& \left.-\frac{\left(\left(z_{i+1}-z_{i}\right) \cdot v_{i}\right)^{2}}{\left|z_{i+1}-z_{i}\right|^{3}}-\frac{\kappa_{1 i}}{2}\left(\frac{z_{i}-z_{i-1}}{\left|z_{i}-z_{i-1}\right|}-\frac{z_{i+1}-z_{i}}{\left|z_{i+1}-z_{i}\right|}\right) \cdot v_{i}\right), \\
l_{i, i+1}^{1}= & \frac{1}{D_{i}}\left(\frac{\left(\left(z_{i+1}-z_{i}\right) \cdot v_{i}\right)\left(\left(z_{i+1}-z_{i}\right) \cdot v_{i+1}\right)}{\left|z_{i+1}-z_{i}\right|^{3}}-\frac{v_{i+1} \cdot v_{i}}{\left|z_{i+1}-z_{i}\right|}-\kappa_{1 i} \frac{\left(z_{i+1}-z_{i}\right) \cdot v_{i+1}}{2\left|z_{i+1}-z_{i}\right|}\right) .
\end{aligned}
$$

At the starting point, if on the $x$-axis, we have set $z_{-1}=\left(x_{1},-y_{1}\right)$, and thus it is easily seen that

$$
\begin{aligned}
\left(z_{1}-z_{0}\right) \cdot v_{0} & =-\left(z_{0}-z_{-1}\right) \cdot v_{0}, \\
\left(z_{1}-z_{0}\right) \cdot v_{1} & =-\left(z_{0}-z_{-1}\right) \cdot v_{-1}, \\
v_{1} \cdot v_{0} & =v_{-1} \cdot v_{0},
\end{aligned}
$$

and hence

$$
l_{01}^{1}=l_{0,-1}^{1} .
$$

As clearly $\kappa_{11}=\kappa_{1,-1}$ we may for the computations replace $l_{01}^{1}$ by twice its value, and we set $l_{0,-1}^{1}=0$.

\subsection{Linearization of the second principal curvature}

As in the previous section we write tildes for the quantities after moving all vertices by $h V_{i} v_{i}$. We also write $v_{i, x}$ for the $x$-coordinate of $v_{i}$, etc. Then formulae (18) and (19) yield

$$
\begin{aligned}
\tilde{\kappa}_{2 i}= & -\frac{1}{2\left(y_{i}+h V_{i} v_{i, y}\right)} \\
& \cdot\left(\frac{x_{i+1}+h V_{i+1} v_{i+1, x}-x_{i}-h V_{i} v_{i, x}}{\left|z_{i+1}+h V_{i+1} v_{i+1}-z_{i}-h V_{i} v_{i}\right|}+\frac{x_{i}+h V_{i} v_{i, x}-x_{i-1}-h V_{i-1} v_{i-1, x}}{\left|z_{i}+h V_{i} v_{i}-z_{i-1}-h V_{i-1} v_{i-1}\right|}\right) .
\end{aligned}
$$

Computing the derivative leads to

$$
\begin{aligned}
& \left.\frac{\mathrm{d}}{\mathrm{d} h} \tilde{\kappa}_{2 i}\right|_{h=0}=-\frac{V_{i} v_{i, y}}{y_{i}} \kappa_{2 i}-\frac{1}{2 y_{i}}\left(\frac{V_{i+1} v_{i+1, x}-V_{i} v_{i, x}}{\left|z_{i+1}-z_{i}\right|}+\frac{V_{i} v_{i, x}-V_{i-1} v_{i-1, x}}{\left|z_{i}-z_{i-1}\right|}\right. \\
& \left.\quad-\frac{x_{i+1}-x_{i}}{\left|z_{i+1}-z_{i}\right|^{3}}\left(z_{i+1}-z_{i}\right) \cdot\left(V_{i+1} v_{i+1}-V_{i} v_{i}\right)-\frac{x_{i}-x_{i-1}}{\left|z_{i}-z_{i-1}\right|^{3}}\left(z_{i}-z_{i-1}\right) \cdot\left(V_{i} v_{i}-V_{i-1} v_{i-1}\right)\right) .
\end{aligned}
$$

Reading off the coefficients of $V_{i-1}, V_{i}$, and $V_{i+1}$ yields $l_{i, i-1}^{2}, l_{i i}^{2}$, and $l_{i, i+1}^{2}$. All the other $l_{i j}^{2}$ are zero. Notice that when $y_{i}=0$ we have $\kappa_{2 i}=\kappa_{1 i}$ and so we simply copy the linearization: $l_{i j}^{2}=l_{i j}^{1}$.

If the starting point is on the $x$-axis, then the linearization of $\kappa_{20}$ is the same as the one of $\kappa_{10}$, because we use $\kappa_{2}=\kappa_{1}$ at the starting point. 


\section{Discretization of the Laplace-Beltrami operator}

\subsection{Discretization of the Laplacian in two dimensions}

If $n=2$ the Laplace-Beltrami operator reduces to taking the second derivative with respect to arc length, $\Delta=\partial_{s s}$. For any function $w$ we discretize this with

$$
\partial_{s s} w\left(z_{i}\right) \approx \frac{1}{D_{i}}\left(\frac{w\left(z_{i+1}\right)-w\left(z_{i}\right)}{\left|z_{i+1}-z_{i}\right|}-\frac{w\left(z_{i}\right)-w\left(z_{i-1}\right)}{\left|z_{i}-z_{i-1}\right|}\right),
$$

where as before $D_{i}=\left(\left|z_{i+1}-z_{i}\right|+\left|z_{i}-z_{i-1}\right|\right) / 2$. That is, we have

$$
\partial_{s s} w\left(z_{i}\right) \approx d_{i, i-1}^{1} w\left(z_{i-1}\right)+d_{i i}^{1} w\left(z_{i}\right)+d_{i, i+1}^{1} w\left(z_{i+1}\right)
$$

with

$$
\begin{aligned}
d_{i, i-1}^{1} & =\frac{1}{D_{i}\left|z_{i}-z_{i-1}\right|}, \\
d_{i i}^{1} & =-\frac{1}{D_{i}}\left(\frac{1}{\left|z_{i+1}-z_{i}\right|}+\frac{1}{\left|z_{i}-z_{i-1}\right|}\right) \\
d_{i, i+1}^{1} & =\frac{1}{D_{i}\left|z_{i+1}-z_{i}\right|} .
\end{aligned}
$$

If the starting point is on the $x$-axis, then because of $z_{-1}=\left(x_{1},-y_{1}\right)$ the formulae are symmetric, so here we replace $d_{01}^{1}$ by twice its value, and set $d_{0,-1}^{1}=0$. Hence

$$
-d_{00}^{1}=d_{01}^{1}=\frac{1}{\left|z_{1}-z_{0}\right|^{2}}, \quad d_{0,-1}^{1}=0 .
$$

The second term in the Laplace-Beltrami operator in three dimensions is equal to the first term because $y_{0}=0$.

\subsection{Discretization of the Laplacian in three dimensions}

For a function $w$ that is radially symmetric on a radially symmetric surface one computes for any parametrization by arc length

$$
\Delta w=\partial_{s s} w+\frac{y^{\prime}}{y} \partial_{s} w
$$

provided $y \neq 0$, and $\Delta w=2 \partial_{s s} w$ if $y=0$ (see the Appendix). Discretization leads to

$$
\frac{y^{\prime}}{y} \partial_{s} w\left(z_{i}\right) \approx \frac{1}{4 y_{i}}\left(\frac{y_{i+1}-y_{i}}{\left|z_{i+1}-z_{i}\right|}+\frac{y_{i}-y_{i-1}}{\left|z_{i}-z_{i-1}\right|}\right)\left(\frac{w\left(z_{i+1}\right)-w\left(z_{i}\right)}{\left|z_{i+1}-z_{i}\right|}+\frac{w\left(z_{i}\right)-w\left(z_{i-1}\right)}{\left|z_{i}-z_{i-1}\right|}\right),
$$

so that as in the previous section one has

$$
\frac{y^{\prime}}{y} \partial_{s} w\left(z_{i}\right) \approx d_{i, i-1}^{2} w\left(z_{i-1}\right)+d_{i i}^{2} w\left(z_{i}\right)+d_{i, i+1}^{2} w\left(z_{i+1}\right)
$$


with

$$
\begin{aligned}
d_{i, i-1}^{2} & =-\frac{1}{4 y_{i}\left|z_{i}-z_{i-1}\right|}\left(\frac{y_{i+1}-y_{i}}{\left|z_{i+1}-z_{i}\right|}+\frac{y_{i}-y_{i-1}}{\left|z_{i}-z_{i-1}\right|}\right), \\
d_{i i}^{2} & =\left(\frac{1}{4 y_{i}\left|z_{i}-z_{i-1}\right|}-\frac{1}{4 y_{i}\left|z_{i+1}-z_{i}\right|}\right)\left(\frac{y_{i+1}-y_{i}}{\left|z_{i+1}-z_{i}\right|}+\frac{y_{i}-y_{i-1}}{\left|z_{i}-z_{i-1}\right|}\right), \\
d_{i, i+1}^{2} & =\frac{1}{4 y_{i}\left|z_{i+1}-z_{i}\right|}\left(\frac{y_{i+1}-y_{i}}{\left|z_{i+1}-z_{i}\right|}+\frac{y_{i}-y_{i-1}}{\left|z_{i}-z_{i-1}\right|}\right) .
\end{aligned}
$$

Of course, if $y_{i}=0$ we set $d_{i j}^{2}=d_{i j}^{1}$, this in particular applies if the starting point is on the $x$-axis.

\section{Adaptive re-meshing}

The evolution tends to move some of the points closer to their neighbors, as compared with other points and their neighbors. It is therefore necessary to every once in a while readjust the position of the points. The idea is to move each point to a location equidistant to its two neighbors. A second, more drastic measure to be taken is the insertion of new points in case points move too far apart, or the removal of points if points cluster too much. The moving to the middle, insertion, and deletion are all done with the same algorithm as described below, so as to preserve the curvature as much as possible.

Let $\tilde{z}_{i}$ be the new location of $z_{i}$, and keep $z_{i+1}$ and $z_{i-1}$ fixed. Then $d_{i}=\left|\tilde{z}_{i}-z_{i-1}\right|=\left|\tilde{z}_{i}-z_{i+1}\right|$. Let $\tilde{v}_{i}$ be the normalized vector $z_{i+1}-z_{i-1}$ turned clockwise by $90^{\circ}$, then $\tilde{z}_{i}=\frac{1}{2}\left(z_{i+1}+z_{i-1}\right)+\epsilon \tilde{v}_{i}$ for some yet to be determined $\epsilon$. For the periodic movement of the points to the middle of their segments, we compute the location of all $\tilde{z}_{i}$ first, and then move them all at once.

Using the equation for $\tilde{z}_{i}$ in (17) we obtain $\kappa_{1 i}=\frac{2 \epsilon}{d_{i}^{2}}$. If $\kappa_{1 i}=0$ then clearly $\epsilon=0$. Otherwise, setting $l_{i}=\left|\frac{1}{2}\left(z_{i+1}-z_{i-1}\right)\right|$, we also have $\epsilon^{2}+l_{i}^{2}=d_{i}^{2}$ by the Pythagorean theorem. Solving these equations for $\epsilon$ yields

$$
\epsilon=\frac{1-\sqrt{1-l_{i}^{2} \kappa_{1 i}^{2}}}{\kappa_{1 i}},
$$

where we have already chosen the minus sign in front of the square root. This choice comes about as follows. Finding the location of $\tilde{z}_{i}$ that preserves the curvature does in fact exactly correspond to finding a circular arc through $z_{i+1}$ and $z_{i-1}$ of prescribed signed curvature. There will be of course two such circular arcs, one shorter than a semi-circle, and one longer than a semi-circle. Choosing a minus sign in the equation for $\epsilon$ above amounts to choosing the shorter arc.

It is certainly possible that we numerically have computed a curvature value $\kappa_{1 i}$ for which no such circular arc can be found (this is the case when $\kappa_{1 i}>1 / l_{i}$ ). In this case the point $z_{i}$ will not be moved.

\section{A test example for the mean curvature flow}

While the main focus of this paper is the application of the proposed scheme to the Willmore flow, we present here our numerical results for the mean curvature flow of a two-dimensional sphere. Because of the radial symmetry the evolution equation $V=-H$ reduces to a separable ordinary 

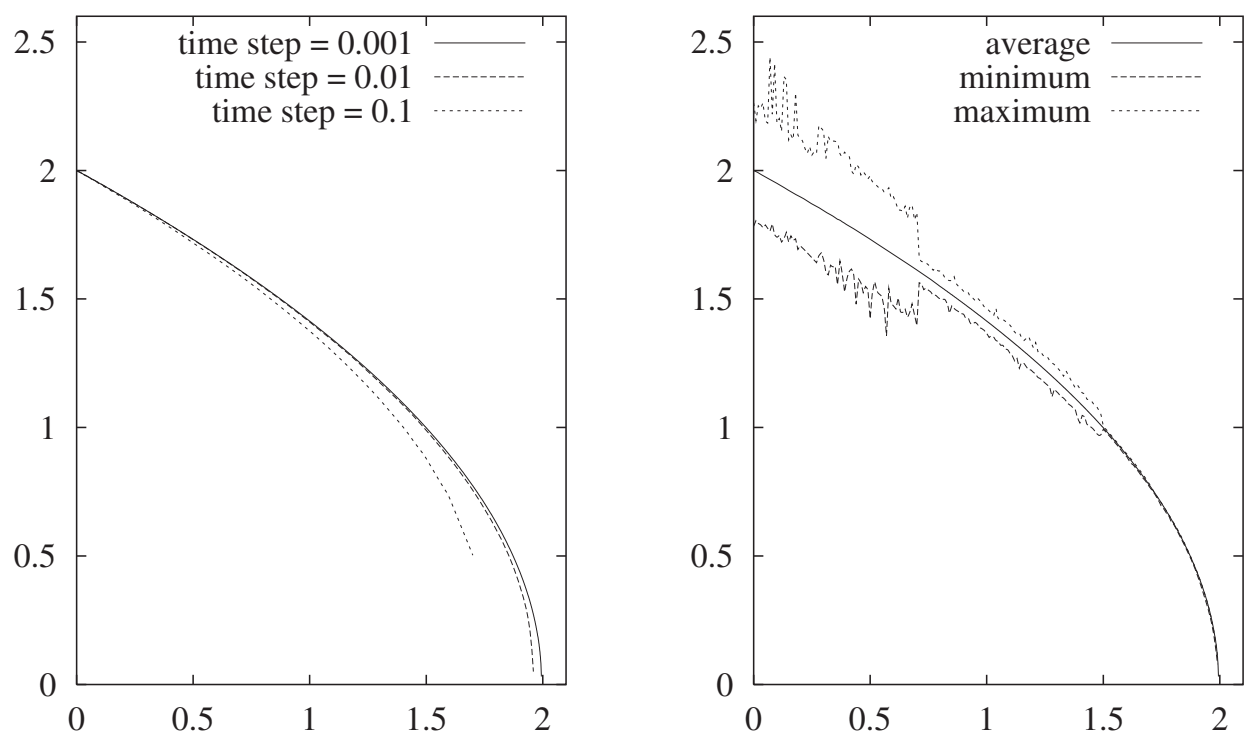

FIG. 1. These curves display the reciprocals of the surface-integral average of the numerical mean curvatures (i.e. $1 / \bar{H}(t)$ ) of the simulation of a sphere driven by the mean curvature flow. The spatial resolution is $0.01-0.05$. The graphs in the panel on the left show the results of varying the time step, where the curve for $\Delta t=0.001$ is essentially visually indistinguishable from the exact solution. The graphs in the panel on the right are for a time step of $\Delta t=0.001$, where we have plotted $\max _{x}(1 / H(x, t)), \min _{x}(1 / H(x, t))$, and $1 / \bar{H}(t)$ as functions of time $t$. See the main text for further description.

differential equation for the radius: $\dot{r}=-1 / r$. Solving this equation leads to

$$
\frac{1}{\bar{H}(t)}=r(t)=\sqrt{r^{2}(0)-2 t} .
$$

We have run a series of computations on this specific problem. We have chosen $r(0)=2$ and a spatial resolution of $0.01-0.05$, and have run the proposed numerical scheme with various time steps. Because of the symmetry only a quarter-circle needs to be considered.

The graphs on the right-hand side of Fig. 1 illustrate several features of the numerical scheme. Considering only the reciprocal of the surface-integral average of the mean curvature is not sufficient to judge the scheme. We thus consider the maximum as well as the minimum of the reciprocal of the discretized mean curvature, and plot these versus time, exhibiting thus the largest deviations of the resulting curve from generating a sphere upon rotation. Note that this results in an exaggerated view of the non-smoothness of the discretized curve, if plotted into a diagram together with an exact quarter-circle, the curves are visually indistinguishable from the quarter-circle. Initially the quartercircle needed for the simulation had 256 points, which for an initial radius of 2 results in a spatial resolution of about 0.012 , well in the mandated range of $0.01-0.05$. In order to save computational cost, we set the simulation to check only every 100 iterations for the allowed spatial resolution range. After 700 time steps, which corresponds to $t=0.7$, the radius had dropped to about 1.613, and the proximity of the points fell under the threshold of 0.01 , so that half of the points got removed. This led to a smoothing of the resulting curve, and is clearly visible in the plot. The same effect happens once more at about $t=1.7$, when the radius falls to about 0.773 , and the proximity of the points falls again under the allowed threshold of 0.01 . 


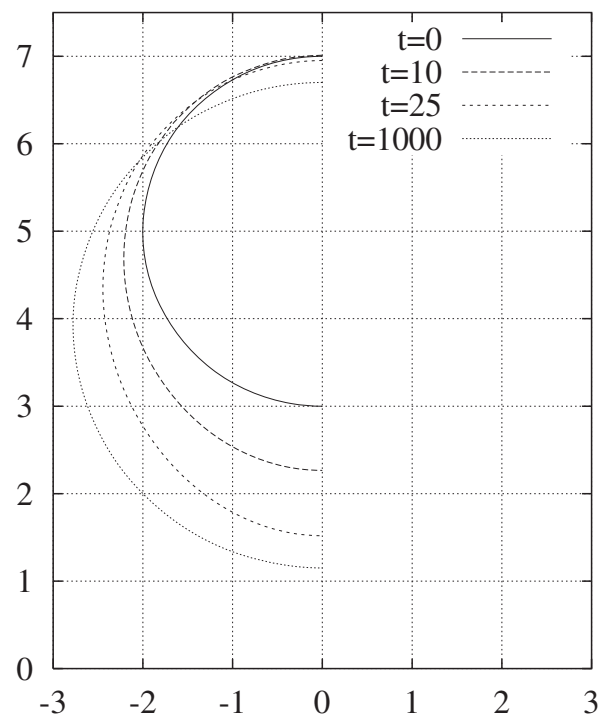

FIG. 2. The generating curves of an evolving torus. Reflect the graphs in the $y$-axis and rotate about the $x$-axis for the full surface.
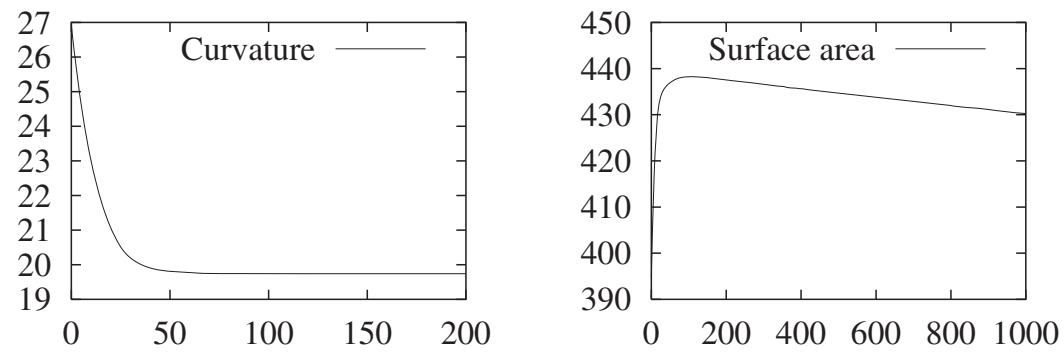

FIG. 3. Left: the total quadratic curvature $\int_{\Gamma(t)} H^{2} \mathrm{~d} \mu$ of an evolving torus. Notice that the graph ends at $t=200$, as to display better the interesting part. Right: the surface area of an evolving torus. The surface area grows initially to decrease the total quadratic curvature.

As can be seen in the plot on the left side of Fig. 1, the numerical scheme is extremely accurate. In fact, for this second-order problem the numerical solution is already a good approximation for a time step equal to the spatial resolution, while one usually expects to need time steps of quadratic magnitude. Also worth pointing out is the good approximation near the singularity. 


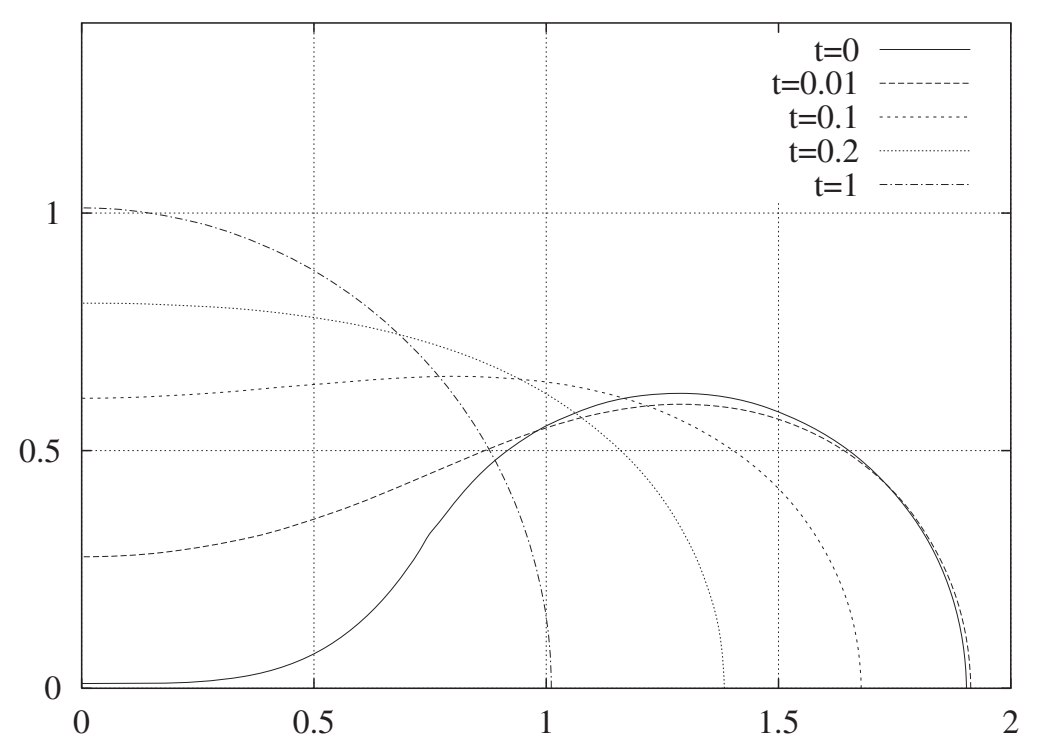

FIG. 4. The generating curves of a dumbbell. Reflect the graphs in the $y$-axis and rotate about the $x$-axis for the full surface. A dumbell does not pinch off under the Willmore flow, but evolves towards a sphere.
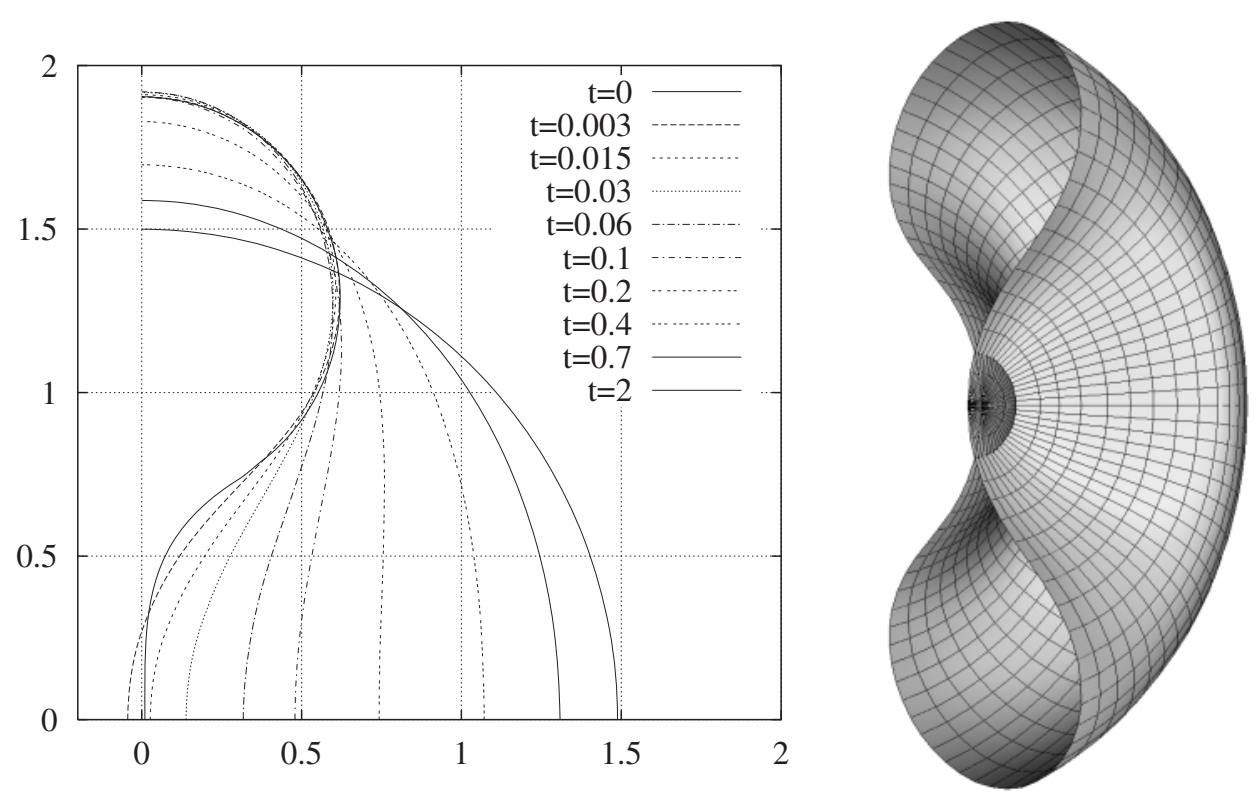

FIG. 5. The graphs on the left show the generating curves of what is initially an erythrocyte; reflection in the $y$-axis and rotation about the $x$-axis generates the full surface. The figure on the right shows half of the generated surface at $t=0.003$. The erythrocyte becomes first immersed, and then evolves towards a sphere. Notice that for $t=0.003$ the generating curve is to the left of the $y$-axis, which upon reflection and rotation results in the non-embeddedness. The non-embeddedness is clearly visible in the center of the figure on the right. 


\section{Numerical experiments for the Willmore flow}

\subsection{A torus}

As mentioned in the introduction, there are stationary tori for the Willmore flow, namely those where the ratio of the two principal radii is $1 / \sqrt{2}$. We chose as starting surfaces several tori that are not stationary. It turned out that the Willmore flow evolves all tori we looked at towards a stationary torus. This is quite in contrast to the surface diffusion flow $(V=\Delta f)$, where a torus cinches the inner hole [16]. Due to the symmetry of the torus we need to use only half of a generating circle to capture the full experiment, see Fig. 2. The time step for the experiment was $10^{-3}$, with spatial distances of the vertices ranging from 0.01 to 0.05 .

Also of interest is the decrease of the total quadratic curvature $\int_{\Gamma(t)} H^{2} \mathrm{~d} \mu$, see (6). The integral levels off at about 19.73878 , which is merely about $-0.02 \%$ away from the theoretical minimum of $2 \pi^{2}$, see Fig. 3. Surface area is not necessarily decreased under the Willmore flow, and for this experiment surface area grows initially in order to decrease $\int_{\Gamma(t)} H^{2} \mathrm{~d} \mu$, see also Fig. 3 .

At $t=1000$ the curvature of the generating curve ranges from 0.33969 to 0.37628 , with an average of 0.36017 , while the $y$-coordinate of the center of mass of the curve is at 3.9404. Using the reciprocal of the average of the plane curvature as the average radius of the generating curve, one obtains $r_{1}=2.776466$, and using the $y$-coordinate of the center of mass as the second radius $r_{2}$ of the torus of revolution, we obtain a ratio of $r_{2} / r_{1}=1.41921$, about $0.35 \%$ from the theoretically established ratio of $\sqrt{2}$ for a stationary Willmore torus. The numerical normal velocity (at $t=1000)$ is less than $7 \times 10^{-5}$. Combined with a time step of $10^{-3}$ this results in a movement of less than $7 \times 10^{-8}$, which is already close to the realm of rounding and discretization errors. On the other hand, the numerical simulation is not quite stationary yet, as can be seen in the still decreasing surface area (see Fig. 3), and at the not yet constant first principal curvature.

\subsection{A dumbbell}

The question arises whether the Willmore flow will like the related surface diffusion flow $(V=\Delta H)$ drive a suitable dumbbell to a pinch-off at the neck [16]. At the neck one certainly has the effects of the surface diffusion flow. On the other hand, $H$ is positive (the smaller the neck, the bigger is $H$ ), and by the arithmetic-geometric inequality we know that $|H| \geqslant|K|$, so that at the neck the term $2 H\left(H^{2}-K\right)$ is positive. The point is, while the $\Delta H$ term can contribute to a movement inwards, it is not expected to overcome the contribution by $2 H\left(H^{2}-K\right)$, which would become positive infinite at a pinching. The numerical solution supports this reasoning: the dumbbells evolve towards the unit sphere. We tested various neck profiles of the shape $y=x^{n}+c, n=1.1,2,4,5$, with $c$ taking a range of (small) values. The graphs in Fig. 4 are for the case of $n=4$ and $c=0.01$, with a time step of $10^{-5}$ and a spatial resolution of $0.01-0.05$.

\subsection{An erythrocyte}

If the curve from the previous section is rotated about its other symmetry axis, one obtains a surface that looks like a red blood cell (i.e. an erythrocyte). Such a surface has been used previously to show that the surface diffusion flow can drive an embedded surface to become immersed [7, 16, 17]. This is also true for the Willmore flow, see Fig. 5. The graphs in Fig. 5 are for a time step of $10^{-6}$ and a spatial resolution of $0.01-0.05$. The initial center profile is given through $y=x^{4}+0.01$, which results in an initial center separation of 0.02 units. 


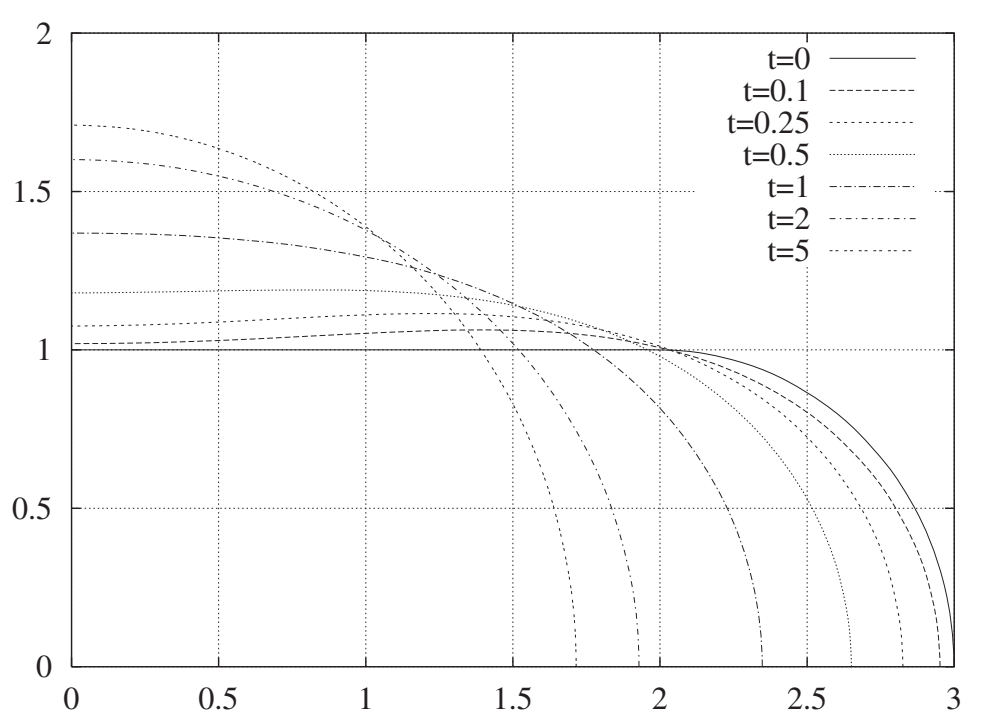

FIG. 6. The generating curves of a tube with spherical end caps; reflection in the $y$-axis and rotation about the $x$-axis generates the full surface. Under the Willmore flow, a (convex) tube becomes first nonconvex, and then evolves towards a sphere.
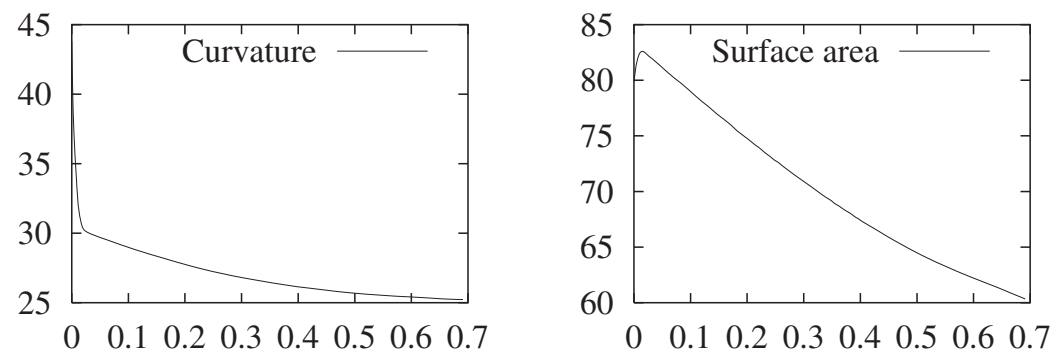

FIG. 7. Left: the total quadratic curvature of the surface evolving towards a singular limit consisting of two spheres. Right: the surface area of the same evolving surface.

\subsection{A tube}

It is known that the surface diffusion flow can evolve convex surfaces into nonconvex ones $[2,8,16]$. This numerical experiment shows that the same is true for the Willmore flow. The graphs in Fig. 6 are for a time step of $10^{-5}$ and a spatial resolution of $0.01-0.05$.

\subsection{A singular example}

It is an open problem whether the Willmore flow can drive a smooth surface to a singularity in finite time. Our numerical experiments seem to indicate that this is the case. For this we take a surface generated by rotation of the part of a limaçon that contains the smaller loop. The Willmore flow pulls that small loop shut. For a related result for the mean curvature flow see [1]. The graphs 


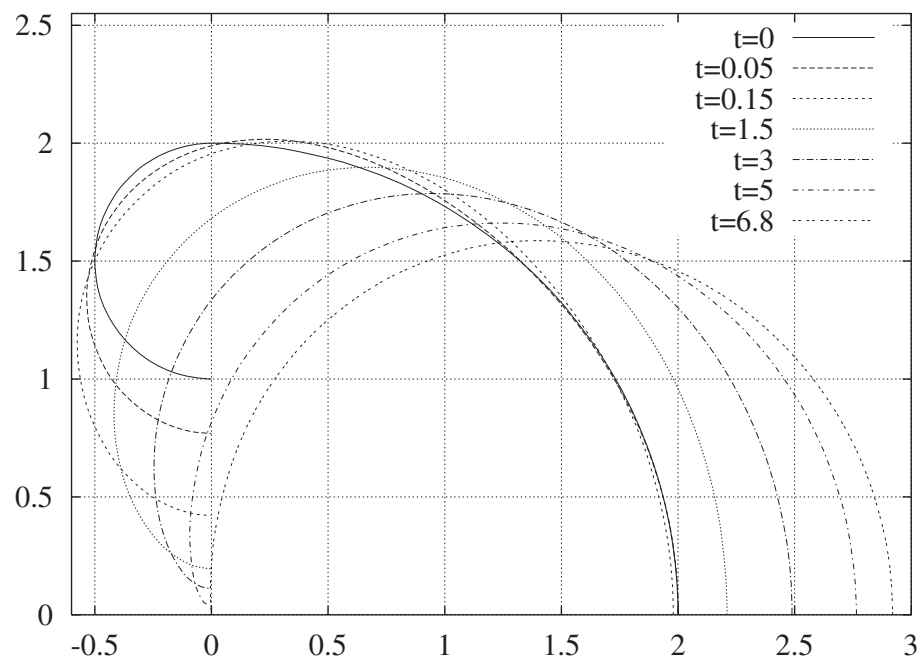

FIG. 8. The generating curves of a surface that develops a singularity under the Willmore flow; reflection in the $y$-axis and rotation about the $x$-axis generates the full surface, see Fig. 9. The Willmore flow pulls the smaller loop shut and creates a singularity. The singular limit consists of two spheres connected tangentially at a single point.

of the generating curves in Fig. 8 are for a time step of $10^{-5}$ and a spatial resolution of $0.01-0.05$. For graphs of the resulting surface see Fig. 9. The figures were displayed using the Geomview package [19], the necessary image format conversions were done with the XV program [3].

The Willmore flow decreases the Willmore energy $\int_{\Gamma(t)} H^{2} \mathrm{~d} \mu$. The initial surface has an energy of about 43.83 , which is more than $8 \pi$, the latter quantity being the total energy of two (disconnected) spheres. This provides the evolving surfaces with an opportunity to move towards a configuration consisting of two spheres. This is indeed what we observe numerically. At the time of the singularity the Willmore energy, that is, the total quadratic curvature, has evolved to about 25.23 , which is about $0.4 \%$ away from $8 \pi$, see Fig. 7. Similarly as for the torus, the surface area grows initially in order to decrease the Willmore energy, see also Fig. 7.

\section{Acknowledgements}

We would like to thank the anonymous referee for helpful suggestions and comments. The research of G. S. has been partially supported by NSF grant DMS-9801337.

\section{REFERENCES}

1. Angenent, S. On the formation of singularities in the curve shortening flow. J. Diff. Geom. 33, (1991) 601-633.

2. Bertozzi, A. L., Bernoff, A. J., \& Witelski, T. P. Axisymmetric surface diffusion: dynamics and stability of self-similar pinch-off. J. Stat. Phys. 93, (1998) 725-776.

3. BRADLEY, J. XV. 1053 Floyd Terrace, Bryn Mawr, PA, USA (1994).

4. BRAKKE, K. E. The surface evolver. Exp. Math. 1, (1992) 141-165.

5. BRyAnt, R. A duality theorem for Willmore surfaces. J. Diff. Geom. 20, (1984) 23-53.

6. Escher, J., MAYER, U. F., \& SimOnett, G. The surface diffusion flow for immersed hypersurfaces. SIAM J. Math. Anal. 29, (1998) 1419-1433. 

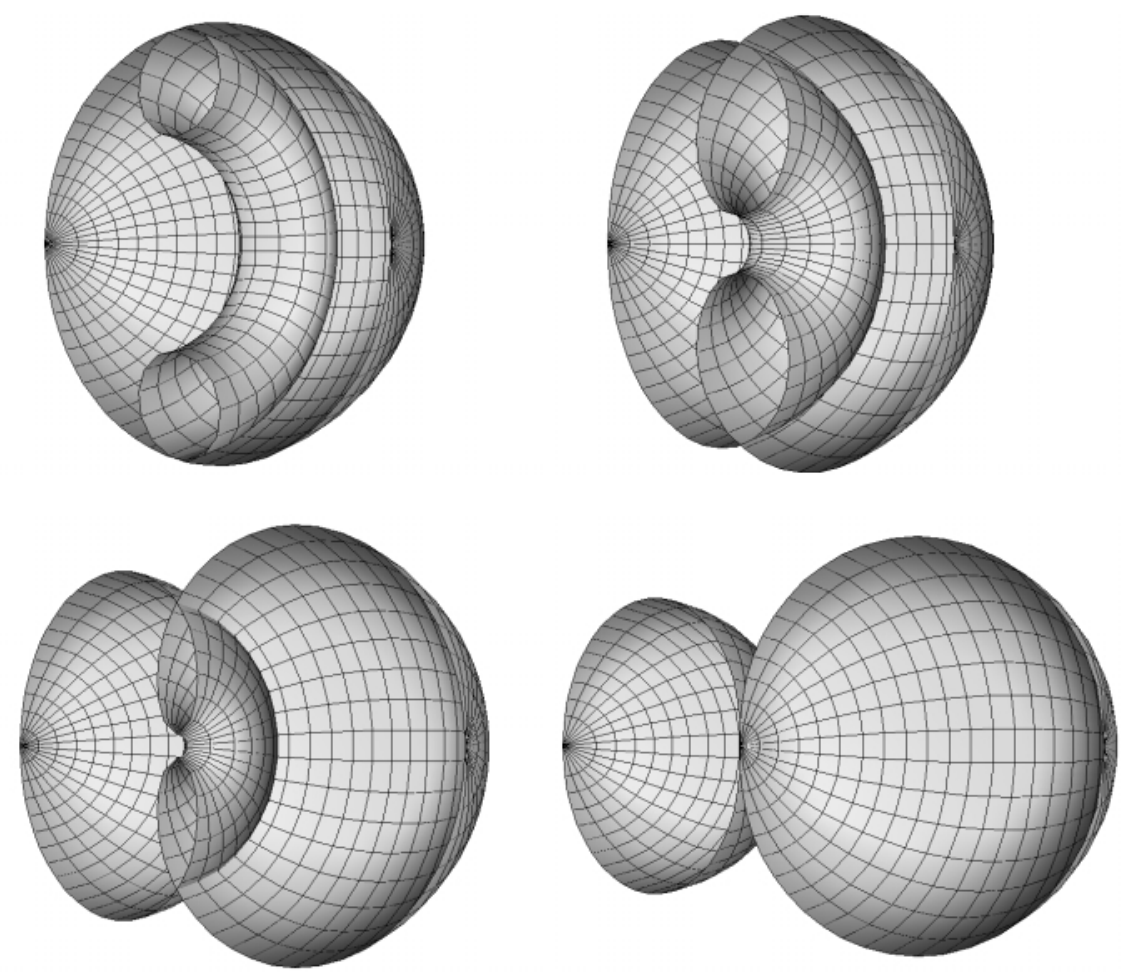

FIG. 9. These figures depict half of the surface generated by the curves of Fig. 8. The computations use more points than the grid on the surface indicates; the grid has been drawn to aid the display only. The initial radii are 0.5 and 2 , respectively. The order is top left at $t=0$, top right at $t=0.05$, bottom left at $t=0.3$, and bottom right at $t=0.68$. The Willmore flow pulls the smaller loop shut and creates a singularity. The singular limit consists of two spheres connected tangentially at a single point.

7. Giga, Y. \& Ito, K. On pinching of curves moved by surface diffusion. Comm. Appl. Anal. 2, (1998) 393-405.

8. GigA, Y. \& ITO, K. Loss of convexity of simple closed curves moved by surface diffusion. In: Escher, J. et al. (ed), Topics in Nonlinear Analysis. The Herbert Amann Anniversary Volume, (Prog. Nonlinear Diff. Equ. Appl. 35). pp. 305-320. Birkhäuser, Basel (1999).

9. Hsu, L., Kusner, R., \& Sullivan, J. Minimizing the squared mean curvature integral for surfaces in space forms. Exp. Math. 1, (1992) 191-207.

10. KUSNER, R. Estimates for the biharmonic energy on unbounded planar domains, and the existence of surfaces of every genus that minimize the squared-mean-curvature integral. In: PETERS, A. K. (ed), Elliptic and Parabolic Methods in Geometry (Minneapolis, MN, 1994). pp. 67-72. Wellesley, MA (1996).

11. Kuwert, E. \& SChÄtzLe, R. Gradient flow for the Willmore functional. Preprint.

12. KuWERT, E. \& SCHÄtZle, R. The Willmore flow with small initial energy. Preprint.

13. LAnger, J. \& Singer, D. Curves in the hyperbolic plane and mean curvatures of tori in 3-space. Bull. London Math. Soc. 16, (1984) 531-534.

14. LI, P. \& YAU, S. T. A new conformal invariant and its applications to the Willmore conjecture and the first eigenvalue of compact surfaces. Invent. Math. 69, (1982) 269-291.

15. MAYER, U. F. A numerical scheme for free boundary problems that are gradient flows for the area functional. Eur. J. Appl. Math. 11, (2000) 61-80. 
16. MAYER, U. F. Numerical solutions for the surface diffusion flow in three space dimensions. Comput. Appl. Math. (2001), accepted.

17. MAYER, U. F. \& Simonett, G. Self-intersections for the surface diffusion and the volume preserving mean curvature flow. Diff. Int. Equ. 13, (2000) 1189-1199.

18. Montiel, S. \& Ros, A. Minimal immersions of surfaces by the first eigenfunctions and conformal area. Invent. Math. 83, (1985) 153-166.

19. Munzner, T., Levy, S., \& Phillips, M. Geomview. The Geometry Center, University of Minnesota, (1994).

20. Pinkall, U. Hopf tori in $S^{3}$. Invent. Math. 81, (1985) 379-386.

21. Pinkall, U. \& Sterling, I. Willmore surfaces. Math. Intelligencer 9, (1987) 38-43.

22. Simon, L. Existence of surfaces minimizing the Willmore functional. Comm. Anal. Geom. 1, (1993) 281-326.

23. Simonett, G. The Willmore flow near spheres. Diff. Int. Equ. 14, (2001) 1005-1014.

24. WeINER, J. L. On a problem of Chen, Willmore et al. Indiana University Math. J. 27, (1978) 19-35.

25. Willmore, T. J. Note on embedded surfaces. An. Şti. Univ. 'Al. I. Cuza' Iaşi Secţ. Ĭa Mat. (NS) B11, (1965) 493-496.

26. Willmore, T. J. Mean curvature of immersed surfaces. An. Ști. Univ. 'Al. I. Cuza' Iași Secț. Ǐa Mat. (NS) 14, (1968) 99-103.

27. Willmore, T. J. Riemannian Geometry. Clarendon, Oxford (1993).

\section{Appendix. Some elementary differential geometry}

Let $s \mapsto(x(s), y(s))$ be a parametrization of a curve by arc length, that is $\left(x^{\prime}(s)\right)^{2}+\left(y^{\prime}(s)\right)^{2}=1$ for all $s$. Assume $y(s)>0$ and $x^{\prime}(s)<0$, so that the curve runs from right to left. This last assumption is not essential, but helps in explaining a few things further down. Rotation of this curve about the $x$-axis results in a surface, with a natural parametrization given as

$$
\phi(s, \theta)=(x(s), y(s) \cos \theta, y(s) \sin \theta) .
$$

In the following we will cease to write the arguments when there is no danger of confusion. We proceed to compute the first fundamental form; for this we compute first

$$
\begin{aligned}
& \phi_{s}=\left(x^{\prime}, y^{\prime} \cos \theta, y^{\prime} \sin \theta\right), \\
& \phi_{\theta}=(0,-y \sin \theta, y \cos \theta) .
\end{aligned}
$$

Hence

$$
\begin{aligned}
& g_{11}=\left\langle\phi_{s}, \phi_{s}\right\rangle=\left(x^{\prime}\right)^{2}+\left(y^{\prime}\right)^{2} \cos ^{2} \theta+\left(y^{\prime}\right)^{2} \sin ^{2} \theta=1, \\
& g_{12}=\left\langle\phi_{s}, \phi_{\theta}\right\rangle=-y y^{\prime} \cos \theta \sin \theta+y y^{\prime} \sin \theta \cos \theta=0, \\
& g_{22}=\left\langle\phi_{\theta}, \phi_{\theta}\right\rangle=y^{2} \cos ^{2} \theta+y^{2} \sin ^{2} \theta=y^{2},
\end{aligned}
$$

and

$$
((g))=\left(\begin{array}{cc}
1 & 0 \\
0 & y^{2}
\end{array}\right), \quad((g))^{-1}=\left(\begin{array}{cc}
1 & 0 \\
0 & \frac{1}{y^{2}}
\end{array}\right), \quad \operatorname{det}((g))=y^{2} .
$$

We proceed to compute the outer normal (to say outer we use $x^{\prime}<0$ and $y>0$ )

$$
\tilde{N}=\phi_{s} \wedge \phi_{\theta}=\left(y y^{\prime},-x^{\prime} y \cos \theta,-x^{\prime} y \sin \theta\right) .
$$


The norm of this vector is

$$
|\tilde{N}|=\left(y^{2}\left(y^{\prime}\right)^{2}+\left(x^{\prime}\right)^{2} y^{2} \cos ^{2} \theta+\left(x^{\prime}\right)^{2} y^{2} \sin ^{2} \theta\right)^{1 / 2}=y,
$$

so that the unit outer normal is given by

$$
N=\left(y^{\prime},-x^{\prime} \cos \theta,-x^{\prime} \sin \theta\right) .
$$

Now we compute the matrix of the second fundamental form $I I=-\langle d N .,$.$\rangle . For this we need$

$$
\begin{aligned}
\phi_{s s} & =\left(x^{\prime \prime}, y^{\prime \prime} \cos \theta, y^{\prime \prime} \sin \theta\right), \\
\phi_{s \theta} & =\left(0,-y^{\prime} \sin \theta, y^{\prime} \cos \theta\right), \\
\phi_{\theta \theta} & =(0,-y \cos \theta,-y \sin \theta) .
\end{aligned}
$$

Using this we obtain the coefficients of $I I$

$$
\begin{aligned}
& h_{11}=-\left\langle\partial_{s} N, \phi_{s}\right\rangle=\left\langle N, \phi_{s s}\right\rangle=x^{\prime \prime} y^{\prime}-x^{\prime} y^{\prime \prime} \cos ^{2} \theta-x^{\prime} y^{\prime \prime} \sin ^{2} \theta=x^{\prime \prime} y^{\prime}-x^{\prime} y^{\prime \prime}, \\
& h_{12}=\left\langle\partial_{s} N, \phi_{\theta}\right\rangle=\left\langle N, \phi_{s}\right\rangle=x^{\prime} y^{\prime} \cos \theta \sin \theta-x^{\prime} y^{\prime} \sin \theta \cos \theta=0, \\
& h_{22}=-\left\langle\partial_{\theta} N, \phi_{\theta}\right\rangle=\left\langle N, \phi_{\theta \theta}\right\rangle=x^{\prime} y \cos ^{2} \theta+x^{\prime} y \sin ^{2} \theta=x^{\prime} y .
\end{aligned}
$$

The matrix of differential of the Gauß map $d N=-((h))((g))^{-1}$ therefore is

$$
\left(\begin{array}{cc}
x^{\prime} y^{\prime \prime}-x^{\prime \prime} y^{\prime} & 0 \\
0 & -\frac{x^{\prime}}{y}
\end{array}\right)
$$

and the two eigenvalues are the two principal curvatures,

$$
\kappa_{1}=x^{\prime} y^{\prime \prime}-x^{\prime \prime} y^{\prime}, \quad \kappa_{2}=-\frac{x^{\prime}}{y} .
$$

Of these two formulae we only use the second one for the computations.

The Laplace-Beltrami operator is defined as $\Delta u=\operatorname{div} \operatorname{grad} u$ for any scalar function $u$ defined on the surface. In local coordinates $\left\{x_{i}: i=1, \ldots, n\right\}$ the gradient is given by

$$
\operatorname{grad} u=\sum_{i k} g^{i k} \partial_{k} u \frac{\partial}{\partial x_{i}}
$$

and for any vector field $X=\sum_{j} X_{j} \frac{\partial}{\partial x_{j}}$ the divergence is defined as

$$
\operatorname{div} X=\sum_{j} \frac{1}{\sqrt{\operatorname{det}((g))}}\left(\partial_{j} \sqrt{\operatorname{det}((g))} X_{j}\right)
$$

Now assume $u=u(s)$ is a function that only depends on the first coordinate, then

$$
\operatorname{grad} u=\left(g^{11} u_{s}, g^{21} u_{s}\right)=\left(u_{s}, 0\right),
$$

and

$$
\Delta u=\operatorname{div} \operatorname{grad} u=\operatorname{div}\left(u_{s}, 0\right)=\frac{1}{\sqrt{\operatorname{det}((g))}}\left(\partial_{s} \sqrt{\operatorname{det}((g))} u_{s}\right)=\frac{1}{y} \partial_{s}\left(y u_{s}\right)=u_{s s}+\frac{y^{\prime}}{y} u_{s} .
$$


This of course is only true if $y \neq 0$. If in fact $y\left(s_{0}\right)=0$ and we have a smooth surface obtained by rotation then

$$
\Delta u\left(s_{0}\right)=2 u_{s s}\left(s_{0}\right) .
$$

This can be seen as follows. By the rotational symmetry one must have $u_{s}\left(s_{0}\right)=0$. The curve must be perpendicular to the $x$-axis and so $y_{s}(s)=1+O\left(s-s_{0}\right)$ and $y(s)=s-s_{0}+O\left(\left(s-s_{0}\right)^{2}\right)$, so that

$$
\lim _{s \rightarrow s_{0}} \frac{y_{s}}{y} u_{s}=\lim _{s \rightarrow s_{0}}\left(1+O\left(s-s_{0}\right)\right) \frac{u_{s}(s)-u_{s}\left(s_{0}\right)}{s-s_{0}+O\left(\left(s-s_{0}\right)^{2}\right)}=u_{s s}\left(s_{0}\right) .
$$

